\title{
MODELO BIOTECNÓLOGICO PARA LA REPRODUCCIÓN DE UNA COLONIA DE CHLORIDEA VIRESCENS
}

\author{
Biotechnological model for the reproduction \\ of a colony of Chloridea Virescens
}

\section{EPISTEMUS}

ISSN: 2007-8196 (electrónico)

ISSN: 2007-4530 (impresa)

Lucía Araceli Manzanarez Jiménez ${ }^{1}$

Recibido: 14/01/2021

Aceptado: 15/03/2021

Publicado: 25/05/2021

DOI: https://doi.org/10.36790/epistemus.v14i28.126

Autor de Correspondencia:

Lucía Araceli Manzanarez Jiménez

Correo:ingenieria_kaizen@hotmail.com

\section{Resumen}

Chloridea virescens es una plaga de importancia económica en el continente americano, la cual es difícil de controlar por presentar resistencia a los insecticidas sintéticos. Por esta razón el objetivo de este estudio fue reproducir una colonia del insecto bajo condiciones de laboratorio mediante un modelo biotecnológico, basado en su ciclo de vida, para mejorar su control. A partir del modelo implementado, los individuos desarrollaron ocho generaciones a lo largo de un año, con ciclo de vida de $44.8 \pm 1.24$ días. Los huevos eclosionaron a los $3.5 \pm 1$ días. Las larvas y las pupas se desarrollaron en $22.4 \pm 1.86$ y $9.2 \pm 2$ días, respectivamente. La superviviencia del adulto fue de $6.7 \pm 1$ días. Este estudio propone el primer modelo biotecnológico para la reproducción de una colonia de $C$. virescens y aporta información valiosa para su reproducción en laboratorio.

Palabras clave: C. virescens, plaga, ciclo de vida.

\section{Abstract}

Chloridea virescens is a pest of economic importance in the american continent, which is difficult to control due to its resistance to synthetic insecticides. For this reason, the objective of this study was to reproduce an insect colony under laboratory conditions using a biotechnological model, based on its life cycle, to improve its control. As of the implemented model, the individuals developed eight generations over a year with a life cycle of 44.8 \pm 1.24 days. The eggs hatched at $3.5 \pm 1$ days. Larvae and pupae developed in $22.4 \pm 1.86$ and $9.2 \pm 2$ days, respectively. Adult survival was $6.7 \pm 1$ days. This study proposes the first biotechnological model for the reproduction of a C. virescens colony and provides valuable information for its reproduction in laboratory..

Keywords: C. virescens, pest, life cycle.

1 Instituto Politécnico Nacional. BEIFI. Centro Interdisciplinario de Investigación para el Desarrollo Integral Regional, Unidad Sinaloa, Blvd. Juan de Dios Bátiz Paredes 250, Col. San Joachín Guasave, Sinaloa, México, Correo Electrónico: ingenieria_kaizen@hotmail.com 


\section{INTRODUCCIÓN}

Chloridea virescens (F.) (Insecta:Lepidoptera: Noctuidae) $=$ Heliothis virescens es un insecto polífago, reportado como plaga principal en el cultivo del algodonero (Gossypium hirsutum L.), y hospedero en tabaco (Nicotiana tabacum L.), tomate (Solanum lycopersicum L.), soya (Glycine max L.) y garbanzo (Cicer arietinum L.) [1], [2], [3]. Las larvas recién emergidas se alimentan de hojas y flores, y a medida que crecen se mueven a las fructificaciones; donde causan perforaciones de aproximadamente $0.5 \mathrm{~cm}$ de diámetro, lo que incrementa el riesgo de enfermedades y afecta en forma directa la calidad de la fibra, principalmente en consistencia y color [2]. Debido a su voracidad, son capaces de causar el $100 \%$ en pérdidas económicas en el algodonero [2]. En tabaco se estiman perdidas por alrededor de $\$ 1,920$ dólares por hectárea [3]. En garbanzo es considerado la plaga de mayor incidencia en el continente americano [4], [5]. El control de las larvas se realiza mayormente con insecticidas químicos sintéticos, cuyo costo representa hasta el $50 \%$ de los costos de producción del cultivo [2].

El ciclo de vida de $C$. virescens tiene una duración estimada de 33-46 días, en consecuencia, durante el ciclo del cultivo puede desarrollar aproximadamente dos generaciones, lo que le permite incrementar sus poblaciones y ocasionar severos daños en el rendimiento del cultivo [4], [5], [9]. Su ciclo estacional inicia de marzo a mayo. Durante el verano pueden ocurrir cuatro o cinco generaciones, seguido por un periodo de hibernación de septiembre a noviembre en estado de pupa [8]. Un aspecto relacionado con la supervivencia de $C$. virescens es la preferencia del insecto por plantas silvestres, las cuales son fuente de alimentación de estas poblaciones que hibernan a través de todo el año [1]. Por esta razón, el conocimiento del ciclo estacional y el ciclo de vida de C. virescens, son importantes, debido a que de su estudio y comprensión se pueden desarrollar estrategias para el control de la plaga mediante el conocimiento de su reproducción.

Una propuesta novedosa que puede contribuir a la comprensión de los aspectos reproductivos de este insecto, es el uso de un modelo biotecnológico para su reproducción en condiciones controladas. Otros autores

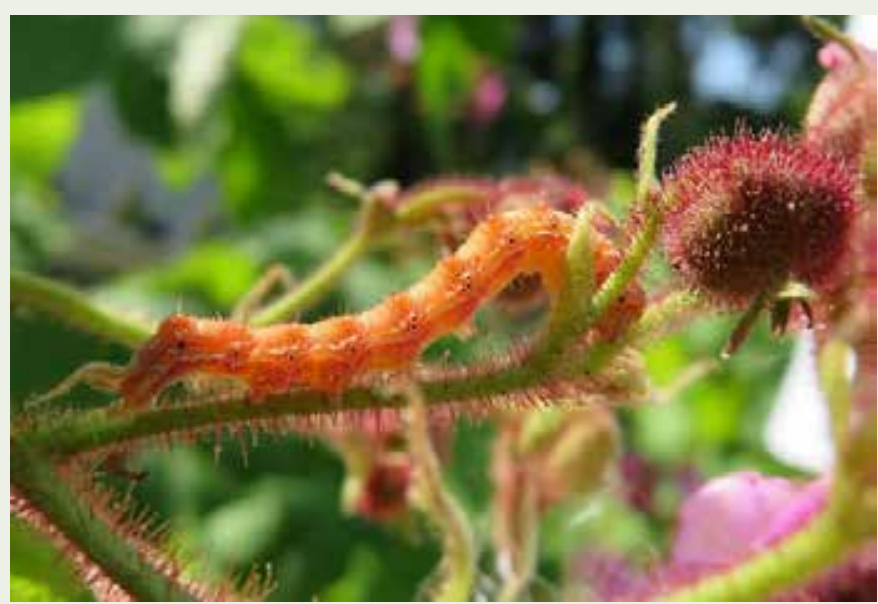

han estudiado su ciclo de vida [4], [5], [9], no obstante, las actividades que involucran la reproducción de un colonia de $C$. virescens, no han sido reportados de manera estructural, por lo que este estudio propone el primer modelo biotecnológico para la reproducción de una colonia de $C$. virescens bajo condiciones de laboratorio.

\section{OBJETIVO}

Las infestaciones de C. virescens en cultivos agrícolas son difíciles de controlar con insecticidas químicos sintéticos, debido a su capacidad para desarrollar resistencia a este tipo de productos [2], [3]. Una alternativa valiosa para el manejo integrado de esta plaga implica conocer aspectos de su reproducción para estimar la duración de los estados de desarrollo de su ciclo de vida, con el fin de diseñar estrategias para su control. Por lo anterior en el presente estudio se validó un modelo biotecnológico para el desarrollo de una colonia de $C$. virescens bajo condiciones de laboratorio, que integra los cuatro estados de desarrollo (Huevo-Larva-Pupa-Adulto) en el ciclo de vida del insecto.

\section{MATERIALES Y MÉTODOS}

El trabajo se efectuó en el período comprendido de febrero de 2018 a marzo de 2019 en el Laboratorio de Bioinsecticidas perteneciente al Centro Interdisciplinario de Investigación para el Desarrollo Integral Regional Unidad Sinaloa (CIIDIR-IPN). Se recolectaron larvas de C. virescens de los últimos instares en cultivos de garbanzo ubicados en Guasave Sinaloa, situado a $25^{\circ} 34^{\prime} 2.8^{\prime \prime} \mathrm{N}$ y $108^{\circ} 28.054^{\prime} \mathrm{O}$ a $15 \mathrm{msnm}$ (Figura 1).

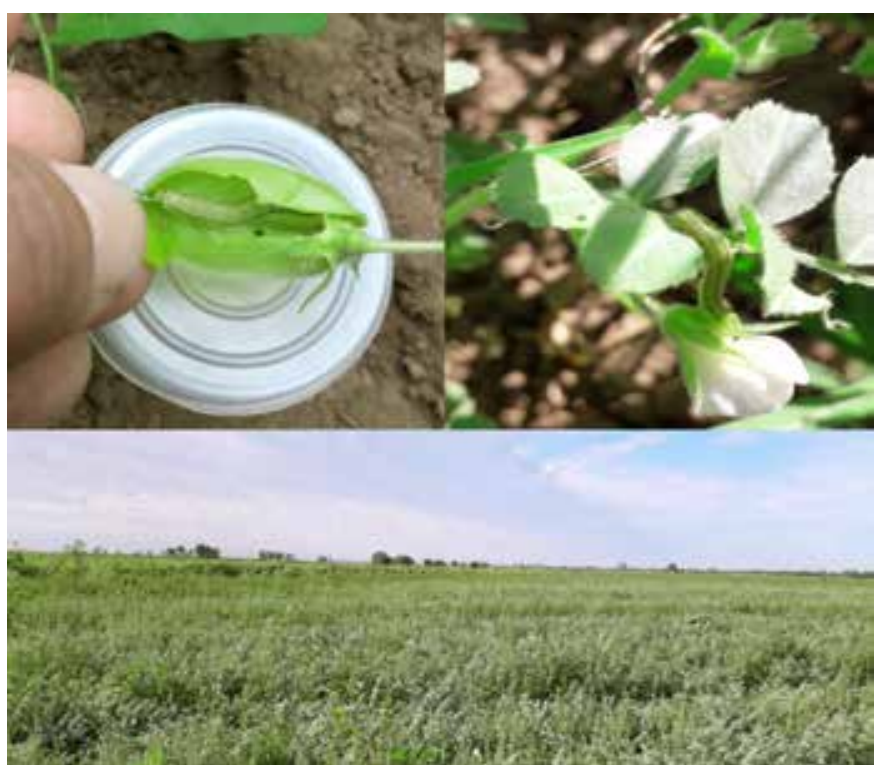

Figura 1. Colecta de larvas en cultivo de garbanzo. a) Larva de último instar (L5: $26 \mathrm{~mm}$ de longitud) de C. virescens colectada en fruto de garbanzo, b) Larva (L5) de $C$. virescens alimentándose de hojas y flores de una planta de garbanzo, c) Cultivo de garbanzo en Guasave Sinaloa. 


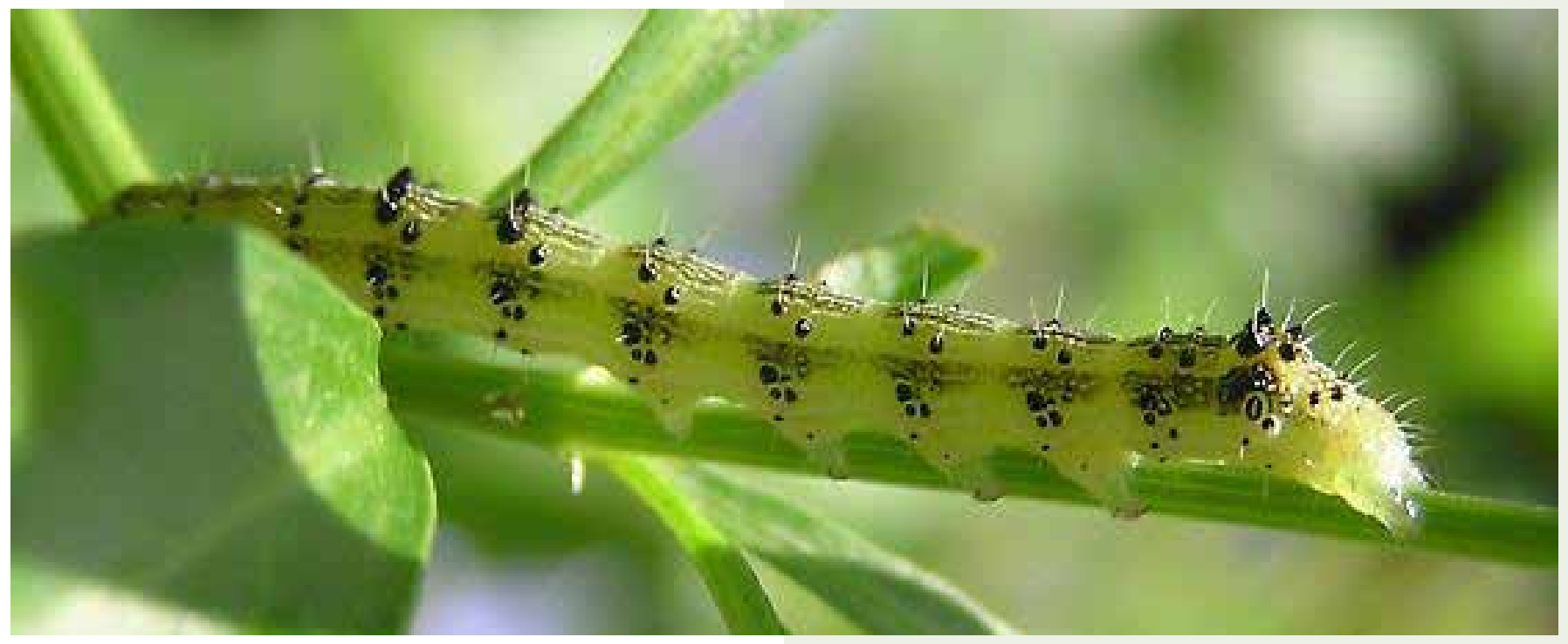

El seguimiento del desarrollo del ciclo de vida de $C$. virescens se hizo mediante un modelo de reproducción con especificaciones biotecnológicas, diseñado a partir de los cuatro estados de desarrollo de C. virescens (Figura 2).

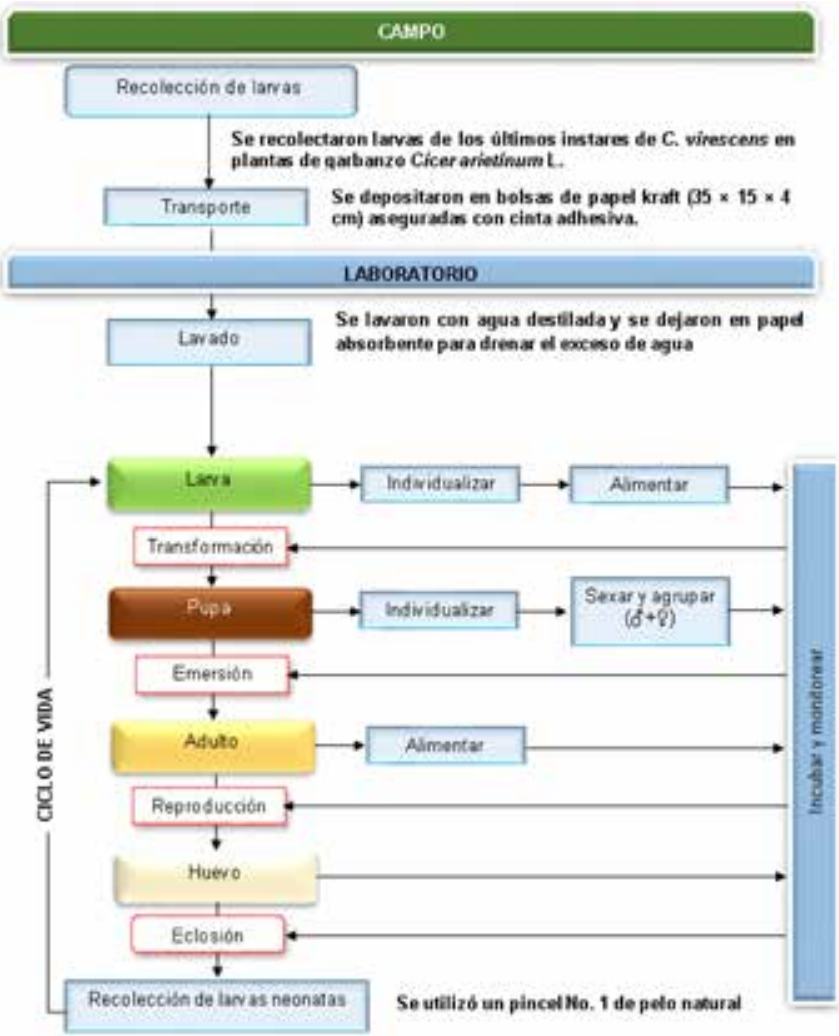
Figura 2. Modelo biotecnológico para la reproducción
de una colonia de C. virescens en laboratorio.

Las larvas recolectadas en campo (200 especímenes) se colocaron individualmente en bolsas de papel para su transporte. En el laboratorio se lavaron con agua destilada para retirar restos de suelo, vegetales y excrementos. Enseguida se individualizaron en recipientes de polipropileno con tapa $(30 \mathrm{ml})$ y se alimentaron con $5-10$ $\mathrm{g}$ de la dieta ICRISAT Diet 3 [10], modificada con aceite de soya, germen de trigo y harina de maíz. En evaluaciones preliminares realizadas en nuestro grupo de investigación con la cría de $C$. virescens, se demostró que el aceite de soya es altamente preferido como un fagoestimulante por las larvas de $C$. virescens. De modo similar, el germen de trigo y la harina de maíz poseen buena palatabilidad para el insecto y proporcionan alta consistencia a la dieta. Además, los tres componentes son fuentes importantes de nutrientes para el desarrollo de las larvas (Tabla 1).

\section{Tabla 1. Dieta artificial para alimentar una colonia de $\mathrm{C}$. virescens}

\begin{tabular}{|l|c|}
\hline \multicolumn{1}{|c|}{ Ingredientes } & Cantidad \\
\hline Harina de maíz & $120 \mathrm{~g}$ \\
\hline Germen de trigo & $55 \mathrm{~g}$ \\
\hline Levadura de cerveza & $35 \mathrm{~g}$ \\
\hline Ácido ascórbico & $3.5 \mathrm{~g}$ \\
\hline Ácido sórbico & $1.1 \mathrm{~g}$ \\
\hline Metilparabeno & $2.2 \mathrm{~g}$ \\
\hline Agar bacteriológico & $15 \mathrm{~g}$ \\
\hline Vitaminas hidrosolubles & $15 \mathrm{ml}$ \\
\hline Aceite de soya & $15 \mathrm{ml}$ \\
\hline Agua destilada & $700 \mathrm{ml}$ \\
\hline
\end{tabular}

Agregar el agar en $500 \mathrm{ml}$ de agua destilada y llevar a punto de ebullición. En una licuadora industrial agregar $200 \mathrm{ml}$ de agua destilada más los ingredientes sólidos y mezclar por 3 min. Enseguida se añade la solución de agar, las vitaminas y el aceite. De nuevo mezclar por 8 min. Finalmente vaciar la mezcla en un molde y tapar. Cuando gelifique cortar en cubos de $1 \mathrm{~cm}^{3}$ o aproximadamente 5-10 g y almacenar en bolsas de cierre hermético a $4^{\circ} \mathrm{C}$. 
La colonia de insectos se mantuvo bajo condiciones controladas en cámara de crecimiento a $28{ }^{\circ} \mathrm{C} \pm 1{ }^{\circ} \mathrm{C}$, humedad relativa de $80 \pm 5 \%$ y fotoperiodo de 14:10 horas de luz:oscuridad. El mantenimiento de la colonia se realizó cada dos días y consistió en limpiar los excrementos y colocar una porción de dieta fresca [11]. Cuando las larvas se transformaron en pupas se pesaron con ayuda de una balanza analítica (Ohaus, Explorer ${ }^{\circledR}$ ), y se midió su longitud en milímetros, utilizando un calibrador digital (UltraTech ${ }^{\oplus}$, Mod. H-7352). Enseguida se sexaron con ayuda de un microscopio estereoscópico (Zeiss Stemi DV4, Oberkochen, Alemania) diferenciando el sexo a partir de las hendiduras de la parte ventral de la pupa [12] con el objetivo de formar parejas (60 $\nabla+60 \bigotimes)$ para la reproducción del insecto. Cada pareja se colocó en una caja de Petri $(60 \mathrm{~mm})$ con una base de algodón humedecido con agua estéril, a continuación, cada caja se colocó en la base de una cámara de emergencia diseñada a partir de un contendor cilíndrico de PVC $(21 \times 15 \mathrm{~cm})$ revestido en su interior con hojas de papel blanco para la oviposición de los huevos y se tapó con película plástica (Parafilm) por ambos extremos.

Al momento de la emergencia de los adultos, se realizó su caracterización mediante claves de Pogue [7]. Los adultos se alimentaron con una solución de miel de abeja (10\%) en agua destilada, para esto, se tomó una caja de Petri $(60 \mathrm{~mm})$ donde se colocó un trozo de algodón y se humedeció con $2 \mathrm{ml}$ de la solución azucarada y se depositó en el fondo del cilindro. Al inicio de la oviposición se contó el número de huevos por contenedor y se midió su tamaño. Además, se observó su forma y la coloración, así como el tiempo de incubación hasta la eclosión de las larvas.

Finalmente, las larvas neonatas se recolectaron con un pincel de pelo natural del No. 01 y se individualizaron. En el desarrollo de las larvas se contó el número y la duración de cada instar observado, también se midió su longitud y el tamaño de la cápsula cefálica, asimismo se realizó la caracterización de larvas con base en la coloración y ornamentación de su cuerpo. Para la segunda generación (F2) se tomaron 120 larvas $(60 \otimes+60 \otimes)$ con el fin de corroborar los datos de cada generación que la colonia produjo durante un año para obtener la validación del modelo. En cada generación se registró la duración de cada estado de desarrollo del insecto.

Los datos obtenidos de las observaciones de crecimiento morfológico y duración de los periodos de desarrollo se analizaron con medidas de tendencia central y dispersión. La información analizada se presentó como promedios más la desviación estándar. El análisis de los datos se realizó con el programa estadístico $S A S^{\circledR}$ version 9.2.

\section{RESULTADOS}

Los adultos obtenidos a partir de las larvas recolectadas en plantas de garbanzo en Guasave Sinaloa fueron identificados como C. virescens, basados en la morfología de la espina protibial, así como el color y la forma de las franjas de sus alas anteriores [7]. Los individuos desarrollaron ocho generaciones (F1-F8) a lo largo de un año y concluyeron su ciclo de vida en $44.8 \pm 1.24$ días, bajo condiciones de laboratorio, siguiendo el modelo de reproducción propuesto.

La hembra de C. virescens depositó $130.5 \pm 13.48$ huevos. La cantidad de huevos a partir de la primera generación (F1) y hasta la última (F8) varió de 8700 a 6780 unidades. A partir de la generación F4, la cantidad de huevos disminuyó $11.11 \%$. En las generaciones siguientes; F5-F6-F7 y F8, la disminución fue de $0.78,3.94,7.88$ y $7.38 \%$, respectivamente. El 


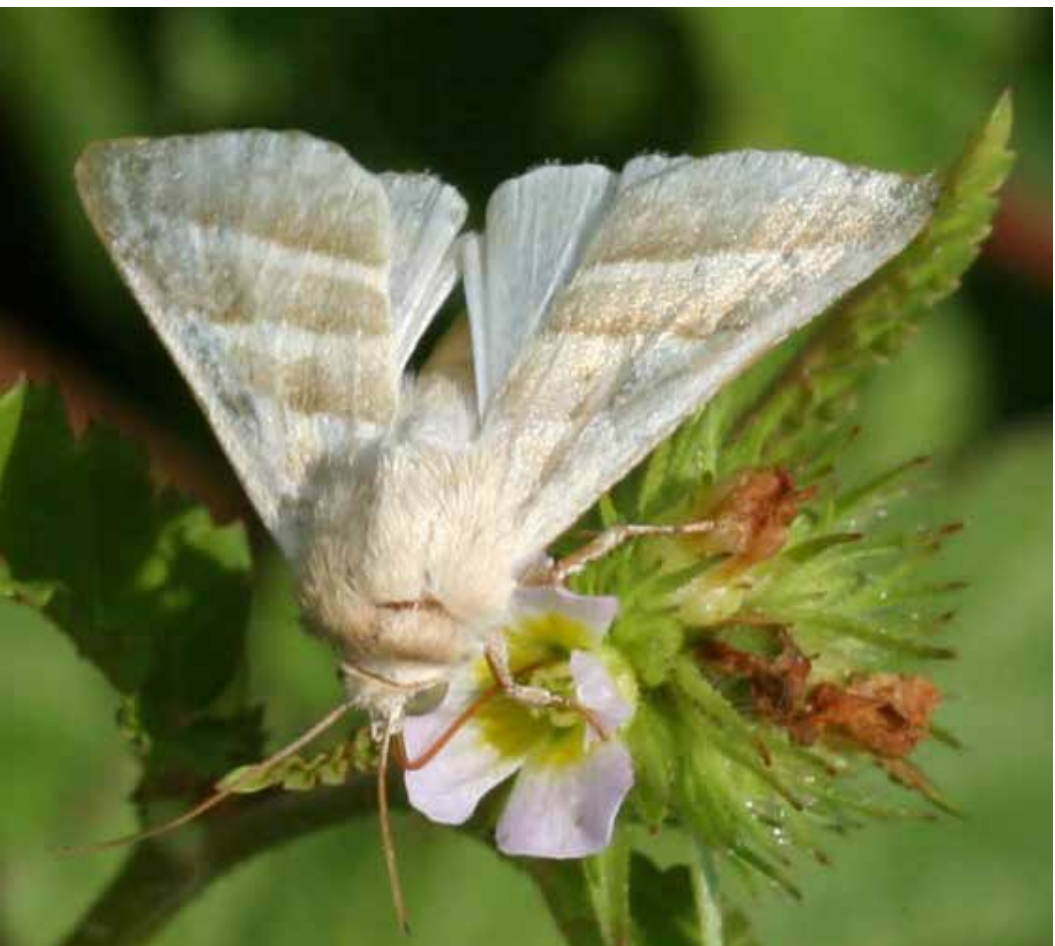

color observado en los huevos fue blanco-crema de forma ovoide con notables estrías longitudinales. Su tamaño midió $0.52 \pm 0.012 \mathrm{~mm}$ de diámetro por $0.50 \pm 0.006 \mathrm{~mm}$ de longitud. El tiempo de eclosión fue de $3.5 \pm 1$ días (Figura 3).

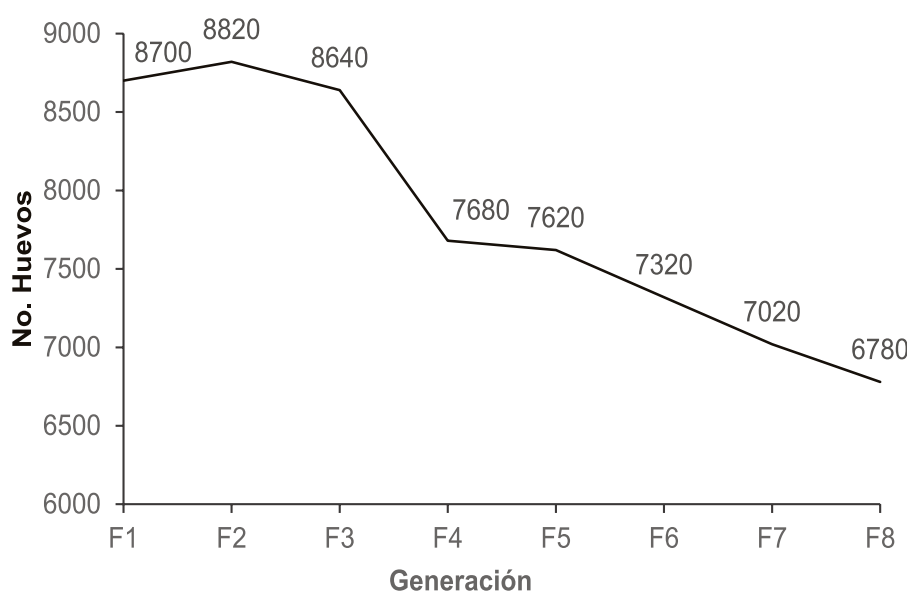

Figura 3. Numero de generaciones y cantidad de huevos de $C$. virescens producidos a lo largo de un año mediante la implementación del modelo biotecnológico para la reproducción de una colonia de c. virescens en de laboratorio.

El tiempo de desarrollo de las larvas fue de $22.4 \pm 1.86$ días. Durante su desarrollo se observaron seis instares, con diferentes medidas de longitud y días de duración (Tabla 2).

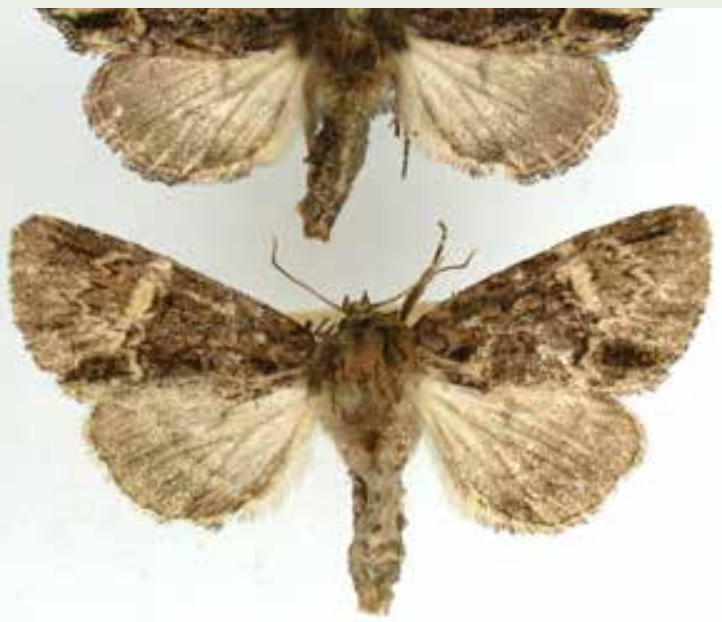

Tabla 2. Longitud de larvas y ancho de las cápsulas cefálicas de cada instar de $C$. virescens y la duración de su tiempo de desarrollo.

\begin{tabular}{|r|c|c|c|}
\hline Instar & $\begin{array}{c}\text { Longitud* }^{*} \\
\text { Media } \pm \text { DE } \\
(\mathbf{m m})\end{array}$ & $\begin{array}{c}\text { Ancho de cápsula } \\
\text { cefálica* } \\
\text { Media } \pm \text { DE }(\mathbf{m m})\end{array}$ & $\begin{array}{c}\text { Duración } \\
\text { (Días) }\end{array}$ \\
\hline L1 & $1.6 \pm 0.005$ & $0.35 \pm 0.011$ & 2.9 \\
\hline L2 & $4.2 \pm 0.010$ & $0.63 \pm 0.008$ & 2.3 \\
\hline L3 & $8.5 \pm 0.008$ & $0.80 \pm 0.013$ & 1.9 \\
\hline L4 & $18.6 \pm 0.012$ & $1.20 \pm 0.004$ & 2.5 \\
\hline L5 & $24.9 \pm 0.018$ & $1.65 \pm 0.009$ & 5.8 \\
\hline L6 & $32.0 \pm 0.021$ & $2.10 \pm 0.001$ & 7.0 \\
\hline
\end{tabular}

* Los datos de la longitud de larvas y el ancho de las cápsulas cefálicas fueron obtenidos con ayuda de un calibrador digital (UltraTech ${ }^{\oplus}$, Mod. H-7352) y un microscopio estereoscópico (Zeiss Stemi DV4, Oberkochen, Alemania).

Las larvas L1 (2.9 días) presentaron coloración blanco hialino con cabeza marrón oscuro, con notables pináculos y sedas en abdomen y tórax. En el instar L2 (2.3 días) fueron capaces de producir pequeños orificios en la dieta artificial. En el instar L3 (1.9 días) se observó cambio en la coloración de manera gradual de blanco hialino a tono marrón oscuro con pronunciamiento de los pináculos y ligera aparición de tres franjas oscuras sub-dorsales y una franja subespiracular de color blanco amarilloso. En este instar la movilidad y la ingesta de dieta fue en aumento, logrando abrir pequeñas cavidades en el sustrato alimenticio. En el instar L4 (2.5 días) las tres franjas oscuras se acentuaron. Además, la ingesta alimenticia fue voraz. Las larvas de los instares L5 y L6 presentaron color marrón claro en el cuerpo, con la cápsula cefálica ligeramente más oscura que el cuerpo. En el instar L5 (5.8 días) sus movimientos fueron más lentos y hubo disminución en la ingesta de alimento. Finalmente, en el instar L6 (7.0 días) eventualmente dejaron de alimentarse y se ubicaron bajo el sustrato alimenticio, donde se trasformaron en pupas. 


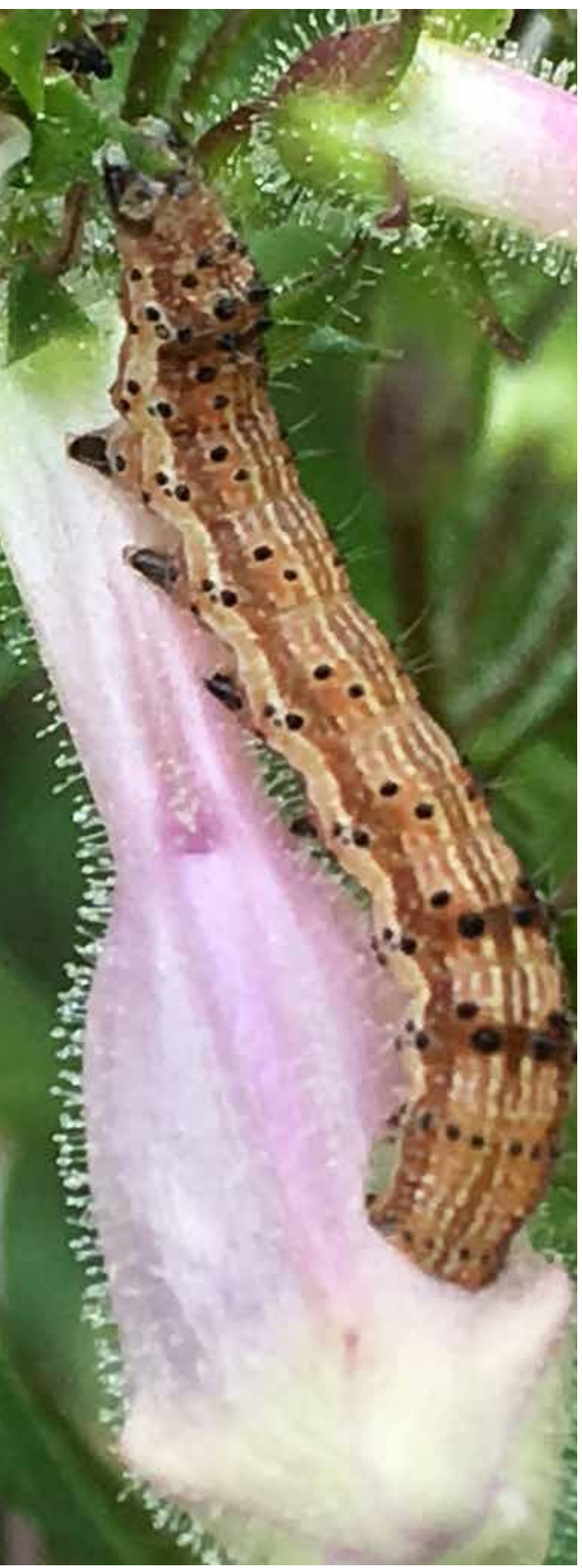

Las pupas pesaron $11.3 \pm 2 \mathrm{~g}$ y midieron $18 \pm 2 \mathrm{~mm}$ de longitud con tiempo de duración de $9.2 \pm 2$ días hasta la emergencia de los adultos.

El adulto se caracterizó por presentar coloración marrónamarilloso, con tres franjas trasversales de color ocre-verde en sus alas anteriores. El periodo de supervivencia de los adultos fue de 6.7 \pm 1 día (Figura 4).

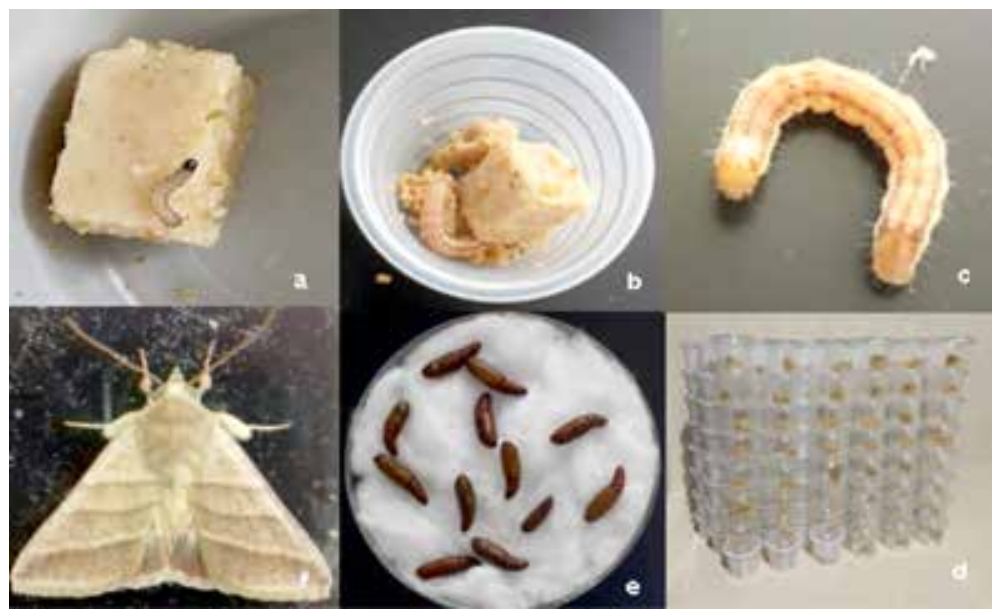

Figura 4. Reproducción de C. virescens mediante un modelo biotecnológico. a) Larva de instar L2; color blanco-hialino con acentuación marrón oscuro en su cabeza. b) Larva de instar L5; alimentándose con dieta artificial. c) Larva de instar L6: tres franjas sub-dorsales acentuadas de color oscuro y sedas notables. d) Larvas en incubación con dieta artificial. e) Pupas incubadas en sustrato de algodón humedecido con agua destilada. f) Adulto de $\mathrm{C}$. virescens; coloración marrónamarilloso, tres franjas transversales de color ocre-verde en las alas anteriores.

\section{DISCUSION}

Para la validación del modelo se tomó como base el ciclo de vida de los individuos de una generación (F1) el cual inicia con la postura de huevos y finaliza cuando las hembras adultas provenientes de dichos huevos depositan sus propios huevos. La población de estudio desarrollo ocho generaciones (F1-F8) en un año. Capinera en 2018, menciona que en condiciones de campo, $C$. virescens produce de cuatro a cinco generaciones durante el verano [8]. Pérez y Suris en 2012, informarón que durante el ciclo del cultivo del garbanzo, esta especie logra desarrollar dos generaciones con posibilidades de incrementar sus poblaciones y generar daños en el rendimiento del cultivo [5]. Esta situación indica el estatus y el potencial del insecto como plaga en cultivos de importancia económica.

En este mismo sentido, el potencial de la plaga es influenciado por la capacidad de las hembras para ovipositar, dado que en condiciones naturales son capaces de producir de 300 a 500 huevos [8]. En nuestros resultados la hembra de $C$. virescens depósito 130.5 \pm 13.48 huevos. Estos resultados difirieron de los reportados por Pérez y Suris en 2012, quienes informaron de $392 \pm 207$ huevos por hembra [5]. Esta diferencia en la cantidad de huevos producida 
podría deberse a la diferencia de tamaño en las cámaras de emergencia utilizadas en nuestro estudio y a la forma del diseño del sustrato para la oviposición de los huevos, la cual se realizó de manera directa en las hojas blancas adheridas a las paredes del cilindro.

A partir de la cuarta generación (F4) se observó disminución en la cantidad de huevos ovipositada. Este fenómeno es atribuido al cruzamiento de progenitores emparentados que ocasionan incremento en la consanguinidad o endogamia [13]. En nuestro estudio, la dismunición de huevos podría deberse al hecho de reproducir la colonia con insectos provenientes de la misma cría de manera constante. García y Bojórquez en 2019, recomiendan continuar la reproducción de la colonia, renovando la descedencia mediante nuevas colectas del insecto, para evitar la endogamia [11]. No obstante, de acuerdo a lo reportado por estos autores, la cantidad de huevos obtenida en este estudio, es adecuada para la reproducción de colonias de insectos utilizados en investigaciones científicas.

La cantidad de instares fue similar a lo informado por Álvarez y colaboradores en 2010 y Méndez en 2003 [4], [9]. Sin embargo, Capinera en 2018, menciona que en condiciones de campo, esta especie puede desarrollar hasta siete instares [8]. En nuestro estudio, durante las generaciones producidas se registró la misma cantidad de instares, lo cual fue corroborado mediante la medición de las cápsulas cefálicas en las larvas [5]. La duración del estado de pupa fue ligeramente menor a lo reportado por Álvarez y colaboradores en 2010 y Méndez en 2003 [4], [ 9]. La diferencia podría deberse al método diseñado en nuestro modelo para incubar las pupas, las cuales fueron colocadas en un sustrato de algodón con humedad superior al $80 \%$, proveída adicionalmente con la atomización de agua destilada cada veinticuatro horas.

Los adultos de $C$. virescens reproducidos en este estudio presentaron las mismas características de ornamentación reportadas previamente por Álvarez et al. en 2010, Pérez y Suris en 2012, Capinera en 2018 y Méndez en 2003 [4], [5], [8], [9], cuyas descripciones coinciden con las informadas por Pogue en 2013 [7].

El estudio de la reproducción de colonias C. virescens bajo condiciones controladas mediante un modelo biotecnológico a partir de su ciclo de vida, parámetros ambientales, alimento artificial y mantenimiento de estándares de reproducción, genera información valiosa para el manejo integrado de esta plaga. Esta información permitiría predecir en qué momento $C$. virescens se presenta en el cultivo, así como cuándo se alimenta, reproduce y oviposita. El conocimiento sobre el momento en que ocurren eventos biológicos importantes de esta plaga es importante para el desarrollo de estrategias de control.

\section{CONCLUSIONES}

Con base en los resultados de este estudio $C$. virescens presentó un ciclo de vida con duración de $44.8 \pm 1.24$ días. Los huevos eclosionaron en $3.5 \pm 1$ días. Las larvas y las pupas se desarrollaron en $22.4 \pm 1.86$ y $9.2 \pm 2$ días, respectivamente. La superviviencia del adulto fue de $6.7 \pm$ 1 días.

El modelo propuesto produjo ocho generaciones a lo largo de un año a partir de larvas recolectadas en campo. La hembra de $C$. virescens depositó $130.5 \pm 13.48$ huevos por cámara de emergencia.

Este estudio propone el primer modelo biotecnológico para la reproducción de $C$. virescens y aporta información para la reproducción de una colonia del insecto para su estudio en condiciones controladas.

\section{BIBLIOGRAFÍA}

[1] J. Molina-Ochoa, W. D. Hutchison, and C. A. Blanco, "Current Status of Helicoverpa zea and Heliothis virescens within a Changing Landscape in the Southern United States and Mexico," Southwest. Entomol., vol. 35, no. 3, pp. 347-354, 2010.

[2] J. Loera-Gallardo, J. I. López-Arroyo, and M. A. Reyes-Rosas, "Complejo Heliothis virescens y Helicoverpa zea (Lepidoptera: Noctuidae)," in Casos de Control Biológico en México, 1a ed., H. C. Arredondo-Bernal and L. A. Rodriguez del Bosque, Eds. México: Dirección General de Sanidad Vegetal, SENASICA, pp. 57-74, 2008.

[3] C. A. Blanco, "Heliothis virescens and Bt cotton in the United States," GM Crop. Food Biotechnol. Agric. Food Chain, vol. 3, no. 3, pp. 201-212, 2012.

[4] U. Álvarez, L. Pérez, M. González, J. Gómez, and J. M. Álvarez, "Biología de Heliothis virescens (Fabricius) en garbanzo (Cicer arietinum L.)," Cent. Agrícola, vol. 37, no. 3, pp. 89-92, 2010.

[5] J. C. Pérez and M. Suris, "Ciclo de vida y reproducción de Heliothis virescens (F.) (Lepidoptera: Noctuidae) sobre garbanzo," Rev. Protección Veg., vol. 27, no. 2, pp. 85-89, 2012.

[6] J. A. Ruiz Corral et al., Plagas de importancia económica en México: Aspectos de su biología y ecología., 1 ra ed., INIFAPCIRPAC-Campo Experimental Centro Altos de Jalisco, p. 459, 2013.

[7] M. G. Pogue, "Revised status of Chloridea Duncan and (Westwood), 1841, for the Heliothis virescens species group (Lepidoptera: Noctuidae: Heliothinae) based on morphology and three genes," Syst. Entomol., vol. 38, pp. 523-542, 2013.

[8] J. L. Capinera, "Tobacco Budworm, Heliothis virescens (Fabricius) (Insecta: Lepidoptera: Noctuidae)," Gainesville, FL 32611, pp. 1-5, 2018.

[9] B. A. Méndez, "Aspectos biológicos sobre Heliothis virescens (Fabricius) (Lepidoptera:Noctuidae) en la empresa municipal agropecuaria Antonio Guiteras de la zona norte de la provincia de las Tunas," Fitosanidad, vol. 7, no. 3, pp. 21-25, 2003.

[10]M. H. Arévalo and I. Zenner de Polanía, “Evaluation of meridic diets suitable for efficient rearing of Heliothis virescens (F.) (Lepidoptera: Noctuidae)," Rev. U.D.C.A Actual. Divulg. Científica, vol. 13, no. 2, pp. 163-173, 2010.

[11]G. C. García and R. C. Bojórquez, Histología y patogenicidad de hongos entomopatógenos, 1 ra ed. México, DF: Instituto Politecnico Nacional, pp. 902019.

[12]M. Walter, "The Life cycle of moths and butterffflies," Rochester Institute of Technology, 1997.

[13]S. H. Tarango-Rivero, "Variación en la historia de vida de dos colonias de Harmonia axyridis Pallas (Coleoptera: Coccinellidae) con diferente tiempo de cría en laboratorio," Folia Entomol. Mex, vol. 107, pp. 51-60, 1999. 